\title{
Sequential sonographic features in neonatal renal vein thrombosis
}

\author{
Aleksandra Mikołajczak ${ }^{1}$, Anna Tytkowska ${ }^{2}$, Agata Jaworska ${ }^{2}$, Alicja Wesołowska ${ }^{2}$, \\ Maria Katarzyna Borszewska-Kornacka ${ }^{3}$, Renata Bokiniec ${ }^{3}$ \\ ${ }^{1}$ Department of Neonatology, Medical University of Warsaw Hospital, Warsaw, Poland \\ ${ }^{2}$ Students Scientific Group affiliated to Department of Neonatology, Medical University of Warsaw, Poland \\ ${ }^{3}$ Neonatal and Intensive Care Department, Medical University of Warsaw, Poland
}

\begin{abstract}
Objectives: Renal vein thrombosis in newborns is a rare but serious and acute disease. Clinical representations of RVT can vary from discrete symptoms to life-threatening conditions. Therefore imaging, and in particular sonography, plays an important role in the diagnosis of RVT in neonates. Gray-scale, color and spectral/power Doppler ultrasound are all used in the diagnosis of RVT.

Material and methods: We present retrospective sequential ultrasonic imaging of three patients (two term and one preterm infant) with findings characteristic of RVT.

Results: Initial ultrasound diagnostic features include: renal enlargement, echogenic medullary streaks, lack of the flow pattern characteristic of arcuate vessels and subsequently loss of corticomedullary differentiation, reduced echogenicity around pyramids and echogenic band at the extreme apex of the pyramid. Higher resistance index or less pulsatile venous flow on the affected kidney are helpful Doppler signs.

Conclusions: Knowledge and identification of specific features of each phase of the evolution of RTV seems essential to prompt diagnosis. We would like to highlight the evolution of specific sonographic features in each subsequent phase of RVT.
\end{abstract}

Key words: neonate, renal vein thrombosis, ultrasound, Doppler imaging

Ginekologia Polska 2018; 89, 5: 271-275

\section{INTRODUCTION}

Renal vein occlusion due to thrombosis is a potentially fatal condition in neonates [1, 2]. It is responsible for 10 to 20 percent of all thrombotic manifestations in the neonatal period [3-5]. Neonates are more susceptible to renal vein thrombosis (RVT) due to immaturity of the hemostatic system, small vessel diameter and underlying diseases $[1,6,7]$.

Thrombosis tends to develop during the first three days after birth but an intrauterine appearance of RTV in the fetus has also been described $[8,9]$. Males are affected more often than females. Left-sided predominance is observed [8]. RVT may be asymptomatic, or present in the form of discrete signs and symptoms, or as a life-threatening condition. The most common clinical triad of findings includes macroscopic hematuria, palpable flank mass and thrombocytopenia $[6,8]$.
However, the classic clinical presentation is only observed in a minority of patients [7].

Diagnosis has been based on a variety of imaging modalities, renal venography being regarded the gold standard while gray scale and Doppler imaging being the most commonly used technique. Ultrasound is less invasive, providing an accessible and reliable method of the evaluation of RVT [3].

The sonographic features vary depending on the severity, the extension of the thrombus, the development of collateral circulation and the stage of renal vein thrombosis. Initial ultrasonic gray-scale diagnostic features - renal enlargement, echogenic medullary streaks and subsequent loss of corticomedullary differentiation - are typically present. An absent, steady or less pulsatile venous flow on the 
affected side as compared with the flow in the contralateral kidney are a helpful Doppler velocimetry finding. After the acute phase, a decrease in echogenicity around the affected pyramids and echogenic band at the extreme apex of the pyramid are revealed $[4,9]$.

\section{Objectives}

To describe the sequential ultrasonic imaging of neonates with findings characteristic of perinatal renal vein thrombosis and the associated risk factors, with emphasis on the clinical characteristics.

\section{MATERIAL AND METHODS}

We retrospectively studied a total of 3 cases of neonatal renal vein thrombosis: the age range of patients at diagnosis was 1-2 nd DOL (day of life), mean -2 nd DOL. We analyzed the type of presentation, the clinical manifestations, and the primarily sequential findings at B-mode and pulsed or power Doppler ultrasonography, as well as the treatment undertaken and the long-term sequelae. All examinations were performed using the Philips HD 15 XE Ultrasound system [Philips N.V. (Royal Philips), Amsterdam, The Netherlands] with a 5-8 $\mathrm{MHz}$ convex probe. Sequential ultrasound features were collected and presented in Table 1.

\section{RESULTS}

Case 1: A male neonate of 40 weeks was born by emergency caesarean section weighing $3710 \mathrm{~g}$. On the first day of life (DOL1), the physical examination revealed a left-sided flank mass in the abdomen. The ultrasound finding on DOL1 showed a left adrenal hemorrhage with enlargement of the left kidney, poor corticomedullary differentiaion and a size discrepancy between the right and the left kidney $(4.08 \mathrm{~cm}$ versus $5.66 \mathrm{~cm}$ ) (Fig. 1). On Doppler velocimetry examination, no venous flow could be demonstrated, spectral Doppler showed abnormal tracings with a narrow peak of the systolic flow and a reversed end-diastolic flow (arrow) indicating increased resistance to flow on DOL1 (Fig. 2).

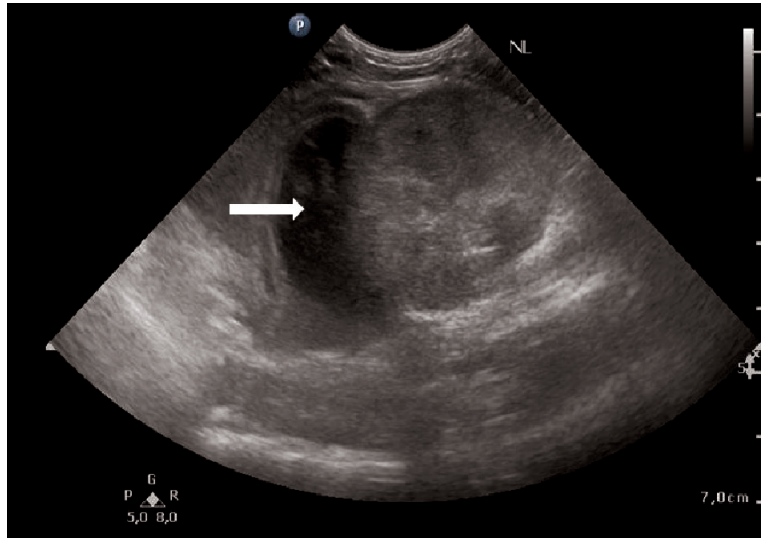

Figure 1. Longitudinal scan of the left flank shows suprarenal mass compatible with adrenal hemorrhage (arrow) on DOL1 in neonate with acute RVT. The image reveals an enlarged, swollen kidney

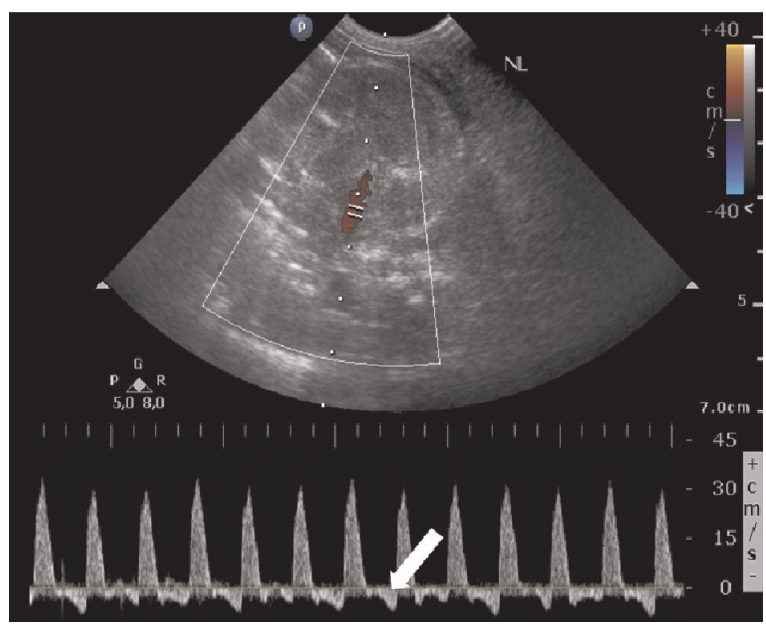

Figure 2. Color and spectral transverse sonogram of the left renal artery on DOL1 demonstrates abnormal spectral tracing with narrow peak of systolic flow, reversed end-diastolic flow (arrow), indicating increased resistance to flow

Follow-up US in the second week (of age) revealed the right kidney involvement in the thrombosis process in the form of a swelling with loss of corticomedullary differentiation.

Table1. Renal vein thrombosis - sequential sonographic features

\begin{tabular}{|c|c|c|c|}
\hline & $\begin{array}{c}\text { Very early acute US features } \\
\text { Less than } 1 \text { week }\end{array}$ & $\begin{array}{l}\text { Still acute US features } \\
\text { The second week }\end{array}$ & $\begin{array}{l}\text { Late US features } \\
\text { The third week and later }\end{array}$ \\
\hline Gray-scale imaging & $\begin{array}{l}\text { - } \text { renal enlargement } \\
\text { - } \text { increase echogenicity } \\
\text { loss of cortico-medullary } \\
\text { differentiation } \\
\text { - echogenic interlobular streaking }\end{array}$ & $\begin{array}{l}\text { - patchwork of hyper-echoic and } \\
\text { hypo-echoic changes (infarct, } \\
\text { hemorrhage, edema) }\end{array}$ & $\begin{array}{l}\text { - } \text { ring of reduced echogenicity } \\
\text { - } \text { calcification } \\
\text { - diffused lace-like echogenicities } \\
\text { or renal atrophy }\end{array}$ \\
\hline Pulsed-Doppler imaging & $\begin{array}{l}\text { - reversed end-diastolic flow in the } \\
\text { main renal artery }\end{array}$ & $\begin{array}{l}\text { - the lack of characteristic flow in } \\
\text { renal vein }\end{array}$ & $\begin{array}{l}\text { recovery of renal flow - color } \\
\text { signal in the main renal vein, } \\
\text { - low resistive index in renal artery }\end{array}$ \\
\hline Power- Doppler imaging & $\begin{array}{l}\text { the lack of characteristic flow } \\
\text { pattern for arcuate vessels }\end{array}$ & & - recovery of arcuate vessel flow \\
\hline
\end{tabular}


A thrombus was found in the inferior vena cava. Spectral Doppler of the affected left kidney showed changes in the resistive index from high to low and decreased perfusion in the second kidney on DOL7. Therapy was started with thrombolysis and heparin. Finally, partial recanalization in the left kidney was obtained. The right kidney became atrophied, resulting in nephrectomy.

Case 2: A newborn male of 37 weeks was born by elective caesarean section, weighing $3620 \mathrm{~g}$. Maternal history included diabetes mellitus. On DOL2 physical examination revealed a left-sided flank mass in the abdomen and left inguinoscrotal bruising. Baseline laboratory tests disclosed thrombocytopenia. Sonogram on DOL2 showed a left adrenal hemorrhage with an edematous left kidney and poor corticomedullary differentiation and streaks - linear hyperechogenicities along the margin of pyramids (Fig. 3A). Color Doppler examination obtained on DOL7 showed absence of vascular flow in the renal vein associated with a thrombus in the left renal vein, turbulence in the left renal artery flow

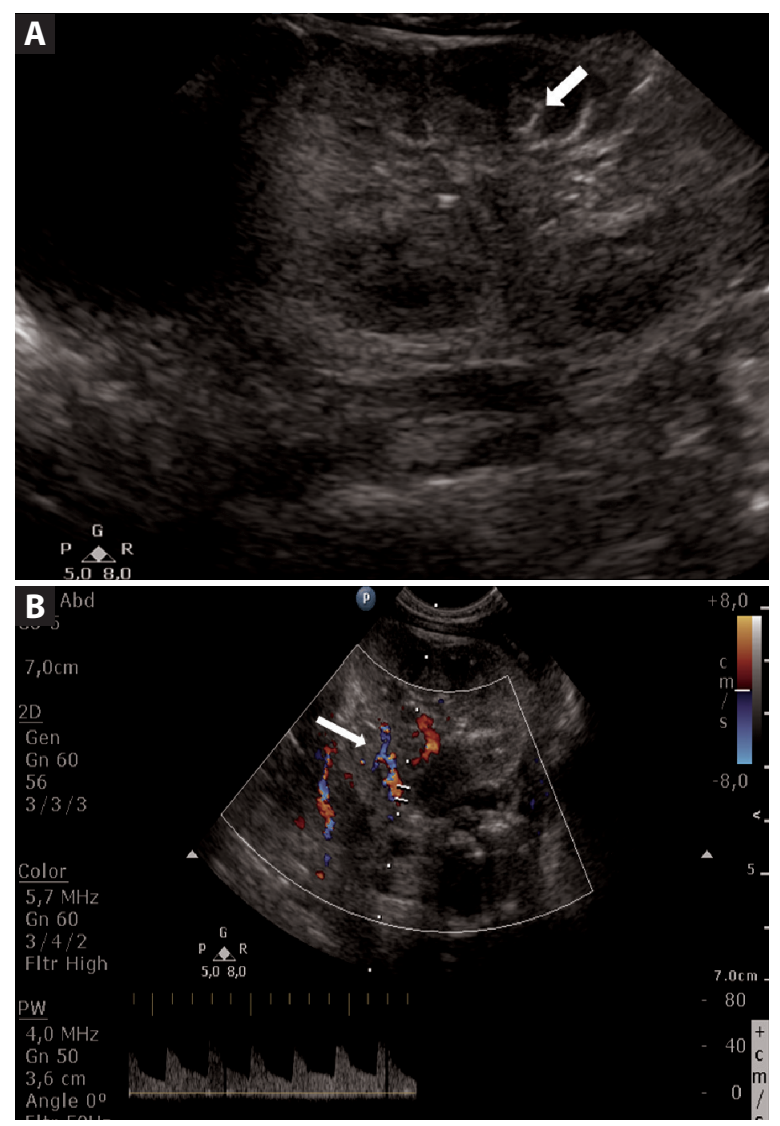

Figure 3. A. US on DOL2 shows loss of corticomedullary differentiation, streaks (arrow) due to the commenced clotting process in peripheral vessels and bleeding in to the adrenal gland. B. Color Doppler on DOL2 of the left artery reveals turbulence in flow connected with low scale, decreased as much as $8 \mathrm{~cm} / \mathrm{s}$ due to poor flow in arterial vessels (arrow). Absence of color signal in the main renal vein. Spectral display presents low resistance to flow connected with aliasing effect, (scale decreased as much as $8 \mathrm{~cm} / \mathrm{s}$ due to poor flow in renal vessels), and showed poor perfusion in arcuate arterial vessels, especially clearly visible on power Doppler imaging (Fig. 3B and Fig. 4A, B). Spectral Doppler reveals normal low resistance to flow in the left renal artery during every phase (Fig. 3B and Fig. 4A). Management with low-molecular-weight-heparin (LMWH) started on DOL4 and was discontinued at three months of age. A follow-up showed the regression of the adrenal changes, recovery of the renal flow but the lace-like linear calcifications remained (Fig. 5).

Case 3: A premature female neonate, weighing $2270 \mathrm{~g}$, as delivered at 34 weeks of gestation by caesarean section following a non-reassuring fetal heart rate. Blood tests revealed thrombocytopenia. On DOL2, US scan showed adrenal hemorrhage, enlargement of the left kidney, loss of corticomedullary differentiation and linear echogenicity along the pyramid margin. Color and spectral Doppler ultrasound revealed no signal flow in the main renal vein, and normal resistive index $(\mathrm{RI}=0.73)$ in the renal artery. There was normal flow in the inferior vena cava. On DOL3 anticoagulant therapy was started. A subsequent follow-up after 8 weeks of therapy showed renal recovery.
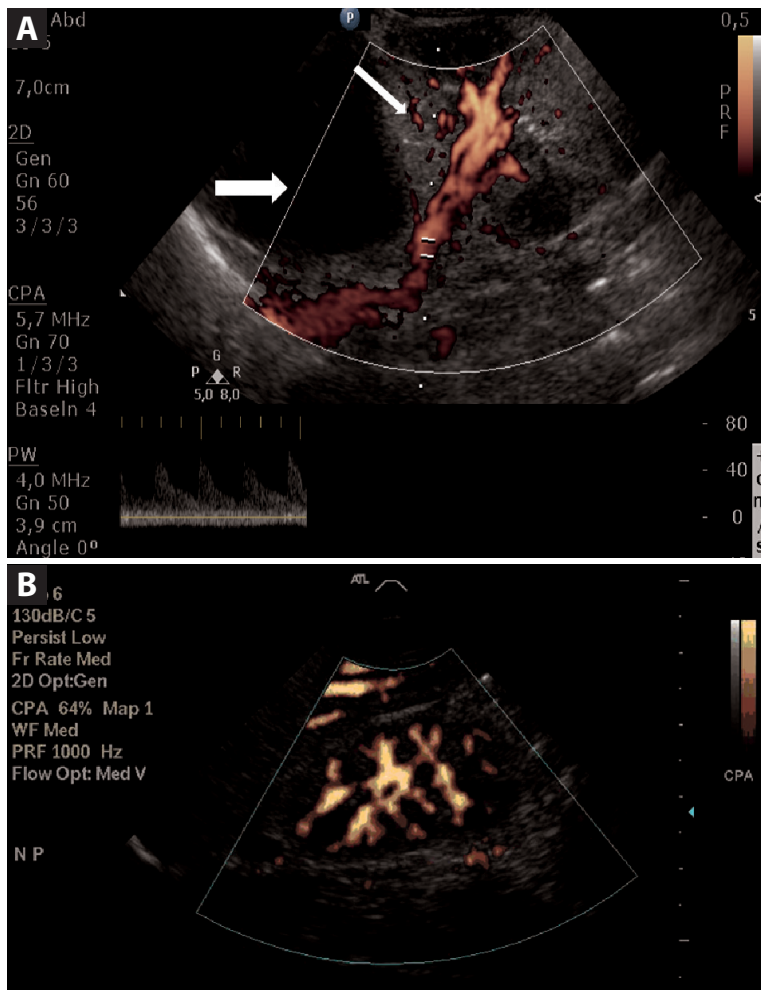

Figure 4. A. A Power Doppler on DOL2 sonogram reveals flow in the main artery with regionally decreased perfusion in peripheral vessels - arcuate arteriae (thin arrow). Suprarenal hypoechoic mass - bleeding in to the adrenal gland (thick arrow). B. Normal perfusion in the right kidney for comparison 


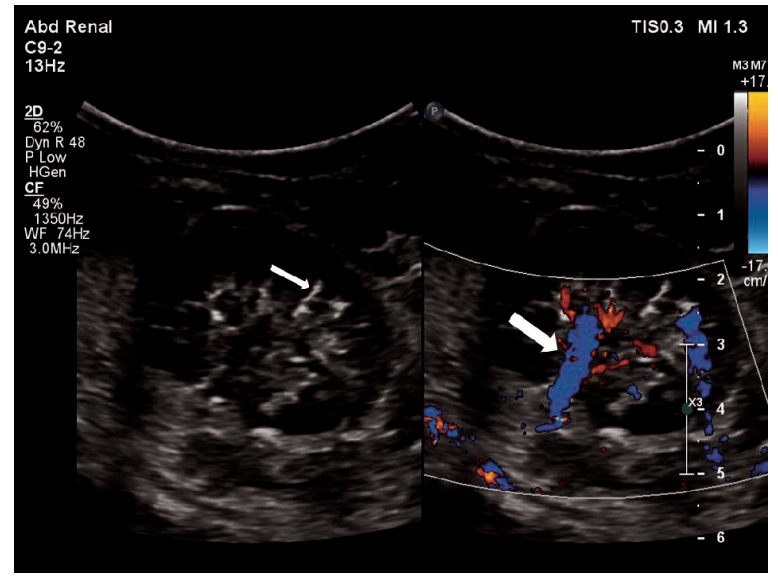

Figure 5. On DOL38 Longitudinal scan of the left kidney shows lace-like and linear calcifications along the margins of the medullary pyramids (thin arrow) consistent with an advanced-stage of RVT. Renal flow recovery in the main renal vein (thick arrow)

\section{DISCUSSION}

To consider US scans of our patients we sum up the sonographic findings variations depending on the extent and duration of RVT. Due to its imprecise criteria, the diagnosis of RVT is not always easy and requires experience from the investigator. The sonographic image may vary and its features may not correlate with age due to the possibility of the clotting process beginning in utero.

It is worth mentioning that renal vein thrombosis begins intrarenally, within small arcuate and interlobular venous vessels [3]. That is why at the early acute stage, using gray-scale ultrasound shows renal enlargement with increased corticoechogenicity and prominent hypoechoic medullary pyramids. The difference in the size of the kidneys provides important diagnostic information at this stage. Echogenic streaks, connected with vascular or perivascular distribution related to interlobular and arcuate thrombi, are also highly indicative of neonatal RVT $[3,4]$. Streaks are first apparent in peripheral vessels due to lack of anastomoses in arcuate veins. They persist only a few days [3]. The investigator should keep in mind that in the first week of the clotting process a fresh thrombus is isoechogenic on gray-scale, that is why color Doppler is required to confirm the presence of flow in the renal vein. As a rule, the clotting process becomes visible after one week. Moreover, the process does not affect both kidneys. In the second week swelling increases with loss of corticomedullary differentiation, echotexture becomes patchy due to areas of hemorrhage and infarction in the renal cortex. At this stage gray-scale ultrasound may demonstrate a thrombus within the renal vein and the IVC because the clotting process originates in the small renal vein and progresses into the main renal vein, ultimately reaching the inferior vena cava. The thrombus extends to the inferior vena cava in about $50 \%$ of cases and adrenal hemorrhage is a complication in $15 \%$ of cases [1, 8]. Adrenal hemorrhage occurs occasionally as a result of an occlusion of the adrenal vein and may be recognized ultrasonically [4]. After two weeks the evolution of the sonographic findings presents puncture or lace-like calcifications [9]. Generally, sequential imaging usually reveals an evolution of sonographic findings [4].

Doppler sonography seems to be the examination of choice for the evaluation of suspected RVT.

At the early phase of RVT Doppler flow findings reveal permanently absent intrarenal and renal venous flow, decreased perfusion in peripheral, arcuate arterial vessels. At this time Power Doppler imaging with very low PRF (Pulse Repetition Frequency) may not depict the flow pattern characteristic of arcuate vessels. Spectral Doppler may show a reversed diastolic flow with an elevated resistive index [3]. A high resistive index of the renal artery flow is not a permanent phenomenon. In Case 1 we observed a high resistance flow in the first week of life, which changed to a low resistance flow with an abnormal-low systolic peak in the next week. It is important to adjust the range of the Doppler velocimetry scale to obtain flow especially when perfusion is decreased. In Case 2 the investigator had to use a scale as low as $8 \mathrm{~cm} / \mathrm{s}$ to measure flow in the renal artery. What the investigator should bear in mind is the phenomenon of the "brain sparing effect" which may result in a high resistance flow in abdominal arteries, the renal artery included, in a newborn during the first hours of life.

Visualizing "streaks" in peripheral vessels should alert the ultrasonographer to the initial phase of renal vein thrombosis and enable the appropriate treatment to be administered $[10,11]$. In our series we conducted the anticoagulant therapy with varying success. In one patient, the outcome was kidney atrophy. We subsequently considered whether therapy should not have been started earlier than on DOL9. In addition, one of our patients recovered completely. In the third patient, the US scan showed small lace-like calcifications in peripheral renal veins. The outcome of these events may vary. The kidney may recover, but the prognosis may as well be poor. Long-term sequelae, such as kidney atrophy, systemic hypertension and chronic kidney disease are common.

\section{CONCLUSIONS}

Ultrasonography has been gaining popularity in diagnosing RVT in neonates. All available gray-scale as well as color Doppler and spectral/power Doppler ultrasound examinations are required to ensure accurate evaluation and monitoring of the process of renal vein thrombosis. We would like to highlight the evolution of specific sonographic features in each subsequent phase of RVT. 


\section{REFERENCES}

1. Resontoc LP, Yap HK. Renal vascular thrombosis in the newborn. Pediatr Nephrol. 2016; 31(6): 907-915, doi: 10.1007/s00467-015-3160-0, indexed in Pubmed: 26173707.

2. Khan OA, Hageman JR, Clardy C. Acute Renal Failure in the Neonate. Pediatr Ann. 2015; 44(10): e251-e253, doi: 10.3928/00904481-2015101210, indexed in Pubmed: 26473428.

3. Brandão LR, Simpson EA, Lau KK. Neonatal renal vein thrombosis. Semin Fetal Neonatal Med. 2011; 16(6): 323-328, doi: 10.1016/j. siny.2011.08.004, indexed in Pubmed: 21865100.

4. Elsaify WM. Neonatal renal vein thrombosis: grey-scale and Doppler ultrasonic features. Abdom Imaging. 2009; 34(3): 413-418, doi: 10.1007/s00261-008-9394-6, indexed in Pubmed: 18404284.

5. Nowak-Göttl U, von Kries R, Göbel U. Neonatal symptomatic thromboembolism in Germany: two year survey. Arch Dis Child Fetal Neonatal Ed. 1997; 76(3): F163-F167, indexed in Pubmed: 9175945.

6. Winyard PJD, Bharucha T, De Bruyn R, et al. Perinatal renal venous thrombosis: presenting renal length predicts outcome. Arch Dis Child Fetal
Neonatal Ed. 2006; 91(4): F273-F278, doi: 10.1136/adc.2005.083717 indexed in Pubmed: 16464938.

7. Kraft JK, Brandão LR, Navarro OM. Sonography of renal venous thrombosis in neonates and infants: can we predict outcome? Pediatr Radiol. 2011;41(3): 299-307, doi: 10.1007/s00247-010-1817-1, indexed in Pubmed: 20734037.

8. Lau KK, Stoffman JM, Williams S, et al. Canadian Pediatric Thrombosis and Hemostasis Network. Neonatal renal vein thrombosis: review of the English-language literature between 1992 and 2006. Pediatrics. 2007; 120(5): e1278-e1284, doi: 10.1542/peds.2007-0510, indexed in Pubmed: 17974721.

9. Wright NB, Blanch G, Walkinshaw S, et al. Antenatal and neonatal renal vein thrombosis: new ultrasonic features with high frequency transducers. Pediatr Radiol. 1996; 26(9): 686-689, indexed in Pubmed: 8781115.

10. Hilario Barrio A, Gallego Herrero C, Miralles Molina M, et al. [Neonatal renal vein thrombosis: early diagnosis using Doppler ultrasonography and long-term sequelae]. Radiologia. 2009; 51(6): 583-590, doi: 10.1016/j. rx.2009.02.008, indexed in Pubmed: 19595392.

11. Moudgil A. Renal venous thrombosis in neonates. Curr Pediatr Rev. 2014; 10(2): 101-106, indexed in Pubmed: 25088263. 